\title{
KINEMATIC WAVE MODELS FOR OVERLAND FLOW*
}

\author{
BY
}

\author{
B. SHERMAN
}

New Mexico Institute of Mining and Technology, Socorro

1. Introduction. We consider the following problem: there is rainfall over a plane rectangular ground area (catchment basin) inclined at a slight angle to the horizontal, and there is infiltration into the ground. The ground is initially dry, the higher end of the ground remains dry at all times, and the rainfall ceases after time $T$. At the bottom end of the rectangle there is a stream or channel into which the ground flow discharges. We will assume that the rainfall and infiltration rate are time-dependent but their space dependency is only on the distance $x$ from the higher edge of the rectangle. It is clear then that the streamlines of the overland flow are lines parallel to the inclined sides of the rectangle and the flow is the same on each streamline. We may ask the following questions: What is the depth $h(x, t)$ and velocity $u(x, t)$ of the flow? Secondly, it is clear that, starting at time $T$, a free boundary $x(t)$ will develop; $x(t)$ is the time history of the water edge as it recedes, because of downflow and infiltration, from the upper edge of the rectangle. What is $x(t)$ ?

An analogous problem can be formulated for a converging catchment basin; this is part of the inner surface of a cone with a very large angle at the vertex. More precisely, let segment $P Q$ of length $L$ intersect a horizontal plant at point $P$. The angle between $P Q$ and the plane is small. Let $P_{1}$ be between $P Q, P_{1} Q=a$. Rotate $P Q$ around the perpendicular to the plane at $P$ through an angle less than $2 \pi$. Then the surface generated by $P_{1} Q$ is the basin. Flow is along the generating segments.

The continuity and momentum equations for these problems are easily formulated [ 1 , chapter 15]; we will obtain the continuity equation for each problem in Sec. 2. It is not possible, in general, to obtain explicit solutions, so it is a question of what reasonable simplifications can be made in order to obtain such solutions. There are two such simplifications. The first is to replace the momentum equation by $u=\alpha h^{m}$, where $m>0$ and $\alpha$ is a friction coefficient which may depend on $x$. The justification for this equation is given in [1, chapter 15]; essentially, these terms are dominant, from the point of view of order of magnitude, in the general momentum equation. The second simplification is to restrict the rainfall and infiltration to be time-independent but still dependent on $x$. Under these conditions explicit solutions can be obtained for both problems, and this is the purpose of the paper. In Sec. 2 we formulate the problem, in Sec. 3 we consider the case infiltration term 0, and in Sec. 4 we consider the case infiltration term not 0 .

The kinematic wave models studied in this paper have been discussed in a number of papers (see references). We have extended the results of those papers by allowing spatial dependency in the rainfall and infiltration rates, in the derivation of the free boundary, and in the unified approach to the two problems described above. There is a concomitant increase in the mathematical complication, but that does not prevent the obtaining of explicit solutions.

* Received June 8, 1976; revised version received November 25, 1976. 
2. Formulation of the problem. We consider first the plane rectangular catchment basin. Let $x$ be the distance from the higher edge, $0 \leq x \leq a$, with $x=a$ the lower edge. Let $q(x, t)$ be the volume of rainfall per unit area per unit time, $f(x, t)$ the volume of infiltration per unit area per unit time, $h(x, t)$ the depth of water, and $u(x, t)$ the velocity, Flow is along lines perpendicular to $x=a$. We assume that, for a specified $T$,

$$
q(x, t)=0, \quad t>T, \quad q(x, t)>f(x, t), \quad 0 \leq t \leq T .
$$

We assume that $f$ depends on $h$ in the following sense:

$$
f(x, t)>0 \quad \text { if } \quad h(x, t)>0 ; \quad f(x, t)=0 \quad \text { if } \quad h(x, t)=0 .
$$

If $d y$ is perpendicular to $d x$ than, referring to the volume of water on the rectangle $d x d y$, we have

inflow: $u h d y d t+q d x d y d t$,

outflow: $\left(u h+(u h)_{x} d x\right) d y d t+\int d x d y d t$,

change in storage: $h_{t} d x d y d t$,

from which we get the continuity equation

$$
h_{t}=(u h)_{x}=q-f .
$$

The momentum equation $[1$, chapter 15$]$ is

$$
Q=u h=\alpha(x) h^{n},
$$

where $n>1$ and $\alpha(x)>0$ measures the surface roughness. From (2.1) and (2.2) we get

$$
h_{t}+\left(\alpha h^{n}\right)_{x}=q-f .
$$

We assume that $h$ is 0 initially, and also at $x=0$ for all $t$ :

$$
h(x, 0)=0, \quad 0 \leq x \leq a, \quad h(0, t)=0, \quad 0 \leq t \leq T .
$$

It is physically plausible that there will be a free boundary $t=t^{0}(x)$ such that, in the semiinfinite strip $D=\{0<x \leq a, t \geq 0\}, h(x, t)=0$ above and on $t=t^{\circ}(x)$ and $h(x, t)>0$ below $t=t^{0}(x) . t=t^{0}(x)$ is the time history of the interface between the covered and uncovered areas of the catchment basin. Thus $h$ is subject to (2.3) in $D$ below $t=t^{\circ}(x)$, and satisfies (2.4) and the condition $h\left(x, t^{0}(x)\right)=0$ for $0 \leq x \leq a$.

Using the same notation for the converging basin, we consider the volume of water on the area $(L-x) d \theta d x$ :

inflow: $u h(L-x) d \theta d t+q(L-x) d x d \theta d t$,

outflow: $\left\{u h(L-x)+[u h(L-x)]_{x} d x\right\} d \theta d t+f(L-x) d x d \theta d t$,

change in storage: $h_{t}(L-x) d x d \theta d t$.

The continuity equation is, therefore,

$$
h_{t}+(u h)_{x}=q-f+\frac{u h}{L-x} .
$$

From (2.5) and (2.2) we get

$$
h_{t}+\left(\alpha h_{n}\right)_{x}=q-f+\frac{\alpha h^{n}}{L-x} .
$$


Again we have the boundary and initial conditions (2.4), and the free boundary $t=t^{0}(x)$.

We will obtain the explicit solutions of problems (2.3)-(2.4) and (2.6)-(2.4) when $q$ and $f$ depend only on $x$. We consider the case $f(x)=0$ in Sec. 3 and the case $f(x) \neq 0$ in Sec. 4 .

3. Infiltration term 0 . In this case $(2.3)$ is

$$
h_{t}+\left(\alpha h^{n}\right)_{x}=q(x)
$$

Taking $x$ as parameter, the characteristic curves are given by

$$
\frac{d t}{d x}=\frac{1}{n \alpha(x) h^{n-1}}, \quad \frac{d h}{d x}=\frac{q(x)-\alpha^{\prime}(x) h^{n}}{n \alpha(x) h^{n-1}} .
$$

The solution of (3.1)-(2.4) is the surface formed by the characteristics passing through $0 \leq$ $x \leq a$ on the $x$-axis and $0 \leq t \leq T$ on the $t$-axis. Thus we add to (3.2) the initial conditions

$$
t\left(x_{0}\right)=0, \quad h\left(x_{0}\right)=0
$$

or

$$
t(0)=t_{0}, \quad h(0)=0
$$

The solution of (3.2)-(3.3) is, indicating dependence on $x_{0}$,

$$
t=t\left(x, x_{0}\right), \quad h=h\left(x, x_{0}\right),
$$

and the solution of (3.2)-(3.4) is

$$
t=t\left(x, t_{0}\right), \quad h=h\left(x, t_{0}\right) .
$$

The curve $t=t(x, 0)$ may (case B) or may not (case A) intersect $t=T$ in $D$. It will be seen, from (3.6) below, that these two cases are distinguished according as

$$
T=\int_{0}^{x} \frac{d \xi}{n \alpha(\xi)^{1 / n} p(\xi)^{(n-1) / n}},
$$

where

$$
p(x)=\int_{0}^{x} q(\xi) d \xi
$$

does (case B) or does not (case A) have a root between 0 and $a$. Since the right side of (3.5) is an increasing function of $x$, (3.5) has no root or exactly one root in $0<x<a$. If $F(x)$ is the right side of (3.5) then $F(a) \leq T$ implies case A and $F(a)>T$ implies case B.

In case $\mathrm{A}, D$ is divided into three regions $D_{1}, D_{2}$, and $D_{3}$; these are the intersections with $D$ of, respectively, $\{t \geq T\},\{t(x, 0) \leq t \leq T\}$, and $\{0 \leq t \leq t(x, 0)\}$. In $D_{2}$

$$
h\left(x, t_{0}\right)=\left[\frac{p(x)}{\alpha(x)}\right]^{1 / n}, \quad t\left(x, t_{0}\right)=t_{0}+\int_{0}^{x} \frac{d \xi}{n \alpha(\xi)^{1 / n} p(\xi)^{(n-1) / n}}
$$

Here $0 \leq t_{0} \leq T$. The curves $t=t\left(x, t_{0}\right)$ do not intersect in $D_{2}, t_{x}\left(x, t_{0}\right)>0$, and $t_{t_{0}}\left(x, t_{0}\right)=$ 1. In $D_{2}$

$$
h(x, t)=\left[\frac{p(x)}{\alpha(x)}\right]^{1 / n}
$$

In $D_{3}$ 


$$
\begin{gathered}
h\left(x, x_{0}\right)=\left[\frac{p(x)-p\left(x_{0}\right)}{\alpha(x)}\right]^{1 / n}, \\
t\left(x, x_{0}\right)=\int_{x_{0}}^{x} \frac{d \xi}{n \alpha(\xi)^{1 / n}\left[p(\xi)-p\left(x_{0}\right)\right]^{(n-1) / n}} .
\end{gathered}
$$

In (3.7) $0 \leq x_{0} \leq a$. By partial integration we get, from (3.7),

$$
t\left(x, x_{0}\right)=\left[\frac{p(x)-p\left(x_{0}\right)}{\alpha(x)}\right]^{1 / n} \frac{1}{q(x)}-\int_{x_{0}}^{x}\left[p(\xi)-p\left(x_{0}\right)\right]^{1 / n} \frac{d}{d \xi} \frac{1}{\alpha(\xi)^{1 / n} q(\xi)} d \xi
$$

We will have $t_{x_{0}}\left(x, x_{0}\right)<0$ provided

$$
\frac{d}{d x} \frac{1}{\alpha^{1 / n} q} \leq 0
$$

or equivalently

$$
\frac{d}{d x}\left(\alpha q^{n}\right) \geq 0
$$

We note that (3.8) includes the case $\alpha(x)$ and $q(x)$ both constant. Thus, under condition (3.8), $t_{x_{0}}\left(x, x_{0}\right)<0$. Also $t_{x}\left(x, x_{0}\right)>0$. The curves $t=t\left(x, x_{0}\right)$ therefore do not intersect in $D_{3}$, and (3.7) defines $h(x, t)$ in $D_{3}$.

To obtain the solution in $D_{1}$ we set $q(x)=0$ in (3.2) and impose initial conditions

$$
t\left(x_{0}^{*}\right)=T, \quad h\left(x_{0}^{*}\right)=\left[\frac{p\left(x_{0}^{*}\right)}{\alpha\left(x_{0}^{*}\right)}\right]^{1 / n}, \quad 0 \leq x_{0}^{*} \leq a .
$$

Then in $D_{1}$

$$
\begin{gathered}
h\left(x, x_{0}{ }^{*}\right)=\left[\frac{p\left(x_{0}^{*}\right)}{\alpha(x)}\right]^{1: n}, \\
t\left(x, x_{0}^{*}\right)=T+\frac{1}{n}\left[p\left(x_{0}^{*}\right)\right]^{-n-1 / n} \int_{x_{0}{ }^{*}}^{x} \alpha(\xi)^{-1 / n} d \xi .
\end{gathered}
$$

It is clear from (3.9) that $t_{x_{0}{ }^{*}}\left(x, x_{0}{ }^{*}\right)<0$, so the curves $t=t\left(x, x_{0}{ }^{*}\right)$ do not intersect in $D_{1}$. For fixed $x t\left(x, x_{0}{ }^{*}\right)$ is defined on the interval $0<x_{0}{ }^{*} \leq x$ and maps that interval in a one to one manner on $t \geq T$. Thus (3.9) defines $h(x, t)$ on $D(T)=\{0<x \leq a, t \geq T\}$. Since, for fixed $x, t \rightarrow \infty$ is equivalent to $x_{0}{ }^{*} \rightarrow 0$, we get $h(x, t) \rightarrow 0$ as $t \rightarrow \infty$. From (3.9) we get

$$
h(x, t)=\frac{\psi(x)}{(t-T)^{1 /(n-1)}}+O\left(t^{-n /(n-1)}\right)
$$

where

$$
\psi(x)=\frac{\left[\int_{0}^{x} \alpha(\xi)^{-1 / n} d \xi\right]^{1 /(n-1)}}{n^{1 / n-1 /} \alpha(x)^{1 / n}}
$$

We note also from (3.9) that $h(x, t)>0$ in $D(T)$ and that, for fixed $t, h(x, t) \rightarrow 0$ as $x \rightarrow 0$. Thus the free boundary $t^{0}(x)$ coincides with the $t$-axis above $t=T$. 
From the above discussion we see that the behavior of $h(x, t)$ for fixed $x$ is as follows: if $(x, t) \in D_{3}$, then, since

$$
h_{t}(x, t)=\frac{h_{x_{0}}\left(x, x_{0}\right)}{t_{x_{0}}\left(x, x_{0}\right)}
$$

$h_{t}(x, t)>0$. If $(x, t) \in D_{2}$ then $h_{t}(x, t)=0$. If $(x, t) \in D_{1}$ then, since (3.10) applies with $x_{0}$ replaced by $x_{0}{ }^{*}, h_{t}(x, t)<0$.

We consider now case B. Let $x^{*}$ be the root of (3.5). Let $D_{11}$ be the part of $D$ above $t=$ $T$ and $t=t(x, 0), D_{12}$ the region bounded by $t=T, t=t(x, 0)$, and $x=a, D_{2}$ the region bounded by $t=T, t=t(x, 0)$, and $x=0$, and $D_{3}$ the part of $D$ below $t=t(x, 0)$ and $t=T$. The solution in $D_{11}$ is given by (3.9), where $0 \leq x_{0}{ }^{*} \leq x^{*}$, the solution in $D_{2}$ is given by (3.6), and the solution in $D_{3}$ is given by (3.7). To obtain the solution in $D_{12}$, let $x_{0}{ }^{*}$ be defined by $T=t\left(x_{0}^{*}, x_{0}\right)$, where $t\left(x, x_{0}\right)$ is given by $(3.7)$ :

$$
T=\int_{x_{0}}^{x_{0}^{*}} \frac{d \xi}{n \alpha(\xi)^{1 / r}\left[p(\xi)-p\left(x_{0}\right)\right]^{(n-1) / n}}, \quad x^{*} \leq x_{0}^{*} \leq a .
$$

We get, in $D_{12}$,

$$
\begin{gathered}
h\left(x, x_{0}\right)=\left[\frac{p\left(x_{0}^{*}\right)-p\left(x_{0}\right)}{\alpha(x)}\right]^{1 / n} \\
t\left(x, x_{0}\right)=T+\frac{1}{n}\left[p\left(x_{0}^{*}\right)-p\left(x_{0}\right)\right]^{-(n-1) / n} \int_{x_{0^{*}}}^{x} \alpha(\xi)^{-1 / n} d \xi .
\end{gathered}
$$

We prove now that $t_{x_{0}}\left(x, x_{0}\right)<0$; this implies that the curves $t=t\left(x, x_{0}\right)$ do not intersect in $D_{12}$, and therefore (3.12) defines $h(x, t)$. A partial integration of (3.11) yields

$$
T=\frac{\left[p\left(x_{0}^{*}\right)-p\left(x_{0}\right)\right]^{1 / n}}{\alpha\left(x_{0}^{*}\right)^{1 / n} q\left(x_{0}{ }^{*}\right)}-\int_{x_{0}}^{x^{*}}\left[p(\xi)-p\left(x_{0}\right)\right]^{1 / n} \frac{d}{d \xi} \frac{1}{\alpha(\xi)^{1 / n} q(\xi)} d \xi .
$$

Differentiating (3.13) with respect to $x_{0}$, we get

$$
\begin{aligned}
0= & \frac{\left[p\left(x_{0}^{*}\right)-p\left(x_{0}\right)\right]^{(1 / n)-1}}{\alpha\left(x_{0}{ }^{*}\right)^{1 / n} q\left(x_{0}{ }^{*}\right)}\left[q\left(x_{0}^{*}\right) \frac{d x_{0}{ }^{*}}{d x_{0}}-q\left(x_{0}\right)\right] \\
& +\int_{x_{0}}^{x *}\left[p(\xi)-p\left(x_{0}\right)\right]^{(1 / n)-1} q\left(x_{0}\right) \frac{d}{d \xi} \frac{1}{\alpha(\xi)^{1 / n} q(\xi)} d \xi .
\end{aligned}
$$

From (3.14) and (3.8) we get

$$
\frac{d x_{0}^{*}}{d x_{0}}>0, \quad q\left(x_{0}^{*}\right) \frac{d x_{0}^{*}}{d x_{0}}-q\left(x_{0}\right) \geq 0,
$$

and therefore

$$
\begin{gathered}
\frac{d}{d x_{0}}\left[p\left(x_{0}^{*}\right)-p\left(x_{0}\right)\right]=q\left(x_{0}^{*}\right) \frac{d x_{0}^{*}}{d x_{0}}-q\left(x_{0}\right) \geq 0, \\
\frac{d}{d x_{0}} \int_{x_{0}^{*}}^{x} \alpha(\xi)^{-1 / n} d \xi<0 .
\end{gathered}
$$

Thus $t_{x_{0}}\left(x, x_{0}\right)<0$. 
The behavior of $h(x, t)$ for fixed $x$ in case $\mathrm{B}$ is the same as in case $\mathrm{A}$ if $0<x \leq x^{*}$; thus $h_{t}(x, t)>0$ if $(x, t) \in D_{3}, h_{t}(x, t)=0$ if $(x, t) \in D_{2}$, and $h_{t}(x, t)<0$ if $(x, t) \in D_{11}$. If $x^{*}$ $<x \leq a$ then the same inequalities apply in $D_{3}$ and $D_{11}$, but in $D_{12}$ we have $h_{t}(x, t) \leq 0$ (since $h_{x_{0}}\left(x, x_{0}\right) \geq 0$ and $\left.t_{x_{0}}\left(x, x_{0}\right)<0\right)$.

We consider now (2.6)-(2.4) when $f=0$. Then

$$
((L-x) h)_{t}+\left((L-x) \alpha h^{n}\right)_{x}=(L-x) q(x) .
$$

If

$$
(L-x) h(x)=\bar{h}(x),(L-x) q(x)=\bar{q}(x), \frac{\alpha(x)}{(L-x)^{n-1}}=\bar{\alpha}(x),
$$

then (3.15) is the same as (3.1). Thus the entire discussion above applies to (3.15)-(2.4), with the appropriate modification of the various expressions for the solutions. Here we have

$$
\bar{p}(x)=\int_{0}^{x} q(\xi)(L-\xi) d \xi .
$$

We note, however, that condition (3.8) becomes

$$
\frac{d}{d x} \alpha(x)(L-x(q) x)^{n} \geq 0 .
$$

This is unsatisfactory since it excludes the case $\alpha(x)$ and $q(x)$ both constant. The situation in $D_{2} \cup D_{1}$ (case A) and in $D_{2} \cup D_{11}$ (case B) is unaffected since (3.17) is not required in order that the curves $t\left(x, t_{0}\right)$ and $t\left(x, x_{0}{ }^{*}\right)$ not intersect. In $D_{3}$ we have, in both cases,

$$
t\left(x, x_{0}\right)=\int_{x_{0}}^{x} \frac{1}{n \alpha(\xi)^{1 / n}}\left[\frac{L-\xi}{p(\xi)-p\left(x_{0}\right)}\right]^{n-1 / n} d \xi .
$$

If $\eta=(L-\xi) /\left(L-x_{0}\right)$,

$$
t\left(x, x_{0}\right)=\int_{\left.(L-x) / L-x_{0}\right)^{\prime}}^{1} \frac{L-x_{0}}{n \alpha\left(L-\left(L-x_{0}\right) \eta\right)^{1 / n}}\left[\frac{\left(L-x_{0}\right) \eta}{\int_{x_{0}}^{L-1 L-x_{0} \mid \eta} q(\xi)(L-\xi) d \xi}\right]^{n-1 / n} d \eta,
$$

and if $\sigma=(L-\xi) /\left(L-x_{0}\right)$,

$$
t\left(x, x_{0}\right)=\int_{(L-x) /\left(L-x_{0}\right)}^{1} \frac{\left(L-x_{0}\right)^{1 / n}}{n \alpha\left(L-\left(L-x_{0}\right) \eta\right)^{1 / n}}\left[\frac{\eta}{\int_{\eta}^{1} q\left(L-\sigma\left(L-x_{0}\right)\right) \sigma d \sigma}\right]^{(n-1 / / n} d \eta .
$$

In order that $t_{x_{0}}\left(x, x_{0}\right)<0$ in $D_{3}$ it is sufficient that $f_{x_{0}}\left(x_{0}, x, \eta\right)<0$, where

$$
f\left(x_{0}, x, \eta\right)=\left[\frac{\left(L-x_{0}\right)}{\alpha\left(L-\left(L-x_{0}\right) \eta\right)}\right]^{1 / n}\left[\int_{\eta}^{1} q\left(L-\sigma\left(L-x_{0}\right)\right) \sigma d \sigma\right]^{-1 n-1 / n} .
$$

If $z=L-\left(L-x_{0}\right) \eta$,

$$
\frac{L-x_{0}}{\alpha\left(L-\left(L-x_{0}\right) \eta\right)}=\frac{L-z}{\eta \alpha(z)} .
$$

Therefore $((L-x) / \alpha(x))^{\prime}<0$ implies 


$$
\frac{\partial}{\partial x_{0}} \frac{L-x_{0}}{\alpha\left(L-\left(L-x_{0}\right) \eta\right)}<0
$$

Also $q^{\prime}(x) \geq 0$ implies

$$
\frac{\partial}{\partial x_{0}} \int_{\eta}^{1} q\left(L-\sigma\left(L-x_{0}\right)\right) \sigma d \sigma \geq 0 .
$$

Thus $((L-x) / \alpha(x))^{\prime}<0$ and $q^{\prime}(x) \geq 0$ imply $f_{x_{0}}\left(x_{0}, x, \eta\right)<0$, and therefore either (3.17) or

$$
\frac{d}{d x} \frac{L-x}{\alpha(x)}<0 \quad \text { and } \quad q^{\prime}(x) \geq 0
$$

implies $t_{x_{0}}\left(x, x_{0}\right)<0$. Condition (3.18) is satisfied when $\alpha(x)$ and $q(x)$ are both constant. Under conditions (3.17) or (3.18) the curves $t=t\left(x, x_{0}\right)$ do not intersect in $D_{3}$.

In regard to $D_{12}$ the curves $t=t\left(x, x_{0}\right)$ do not intersect under condition (3.17), according to our earlier discussion. We show that the same is true under condition (3.18). We have, in $D_{12}$,

$$
\begin{aligned}
t\left(x, x_{0}\right) & =T+\int_{x_{0}^{*}}^{x} \frac{(L-\xi)^{(n-1) / n}}{n \alpha(\xi)^{1 / n}} d \xi\left[\int_{x_{0}}^{x_{0}^{*}} q(\xi)(L-\xi) d \xi\right]^{-(n-1) / n}, \\
T & =\int_{x_{0}}^{x_{0}^{*}} \frac{(L-\xi)^{(n-1) / n}}{n \alpha(\xi)^{1 / n}}\left[\int_{x_{0}}^{\xi} q(\xi)(L-\xi) d \xi\right]^{-(n-1) / n} d \xi
\end{aligned}
$$

These equations become, on introducing $\eta$ and $\sigma$ as above,

$$
\begin{gathered}
t\left(x, x_{0}\right)=T+\frac{1}{n} \int_{(L-x) /\left(L-x_{0}\right)}^{z}\left[\frac{L-x_{0}}{\alpha\left(L-\left(L-x_{0}\right) \eta\right)}\right]^{1 / n} \\
\cdot d \eta\left[\int_{z}^{1} q\left(L-\left(L-x_{0}\right) \eta\right) \eta d \eta\right]^{-(n-1) / n}, \\
n T=\int_{z}^{1}\left[\frac{L-x_{0}}{\alpha\left(L-\left(L-x_{0}\right) \eta\right)}\right]^{1 / n}\left[\int_{\eta}^{1} q\left(L-\left(L-x_{0}\right) \sigma\right) \sigma d \sigma\right]^{-(n-1) / n} \eta^{(n-1) / n} d \eta,
\end{gathered}
$$

where $z=\left(L-x_{0}{ }^{*}\right) /\left(L-x_{0}\right)$. From the second equation it is clear that $d z / d x_{0}<0$. Then, from the first equation, $t_{x_{0}}\left(x, x_{0}\right)<0$.

The expressions for the solution of (3.15)-(2.4) may be obtained from the solution of (3.1)-(2.4) by using (3.16).

4. Infiltration term not 0 . We consider first

$$
h_{t}+\left(\alpha h^{n}\right)_{x}=q(x)-f(x),
$$

together with (2.4). The characteristic curves are

$$
\frac{d t}{d x}=\frac{1}{n \alpha(x) h^{n-1}}, \quad \frac{d h}{d x}=\frac{q(x)-f(x)-\alpha^{\prime}(x) h^{n}}{n \alpha(x) h^{n-1}},
$$

and the initial conditions are (3.3) or (3.4). We now have three cases depending on the relative disposition of $t=t^{0}(x), t=T$, and $t=t(x, 0)$ :
A. $t^{0}(x)>T>t(x, 0), \quad 0<x \leq a$.

$\mathrm{B}_{1} . t^{0}(x)>T$ and $t^{0}(x)>t(x, 0)$, but $t=T$ and $t=t(x, 0)$ intersect at $x^{*}$, i.e., $T=t\left(x^{*}\right.$, $0)$ and $0<x^{*}<a$. 
$\mathrm{B}_{2} \cdot t^{0}(x)>T$, but $t=T$ and $t=t(x, 0)$ intersect at $x=x^{*}$ and $t=t^{0}(x)$ and $t=t(x$, $\left.x^{*}\right)\left(t=t\left(x, x^{*}\right)\right.$ is the prolongation $t=t(x, 0)$ to the right of $\left.x=x^{*}\right)$ intersect at $x=\bar{x}$, i.e., $t^{0}(\bar{x})=t\left(\bar{x}, x^{*}\right)$ and $0<\bar{x}<a$.

These cases can be distinguished prior to the solution of (4.1)-(2.4). We have case A when

$$
T=\int_{0}^{x} \frac{d \xi}{n \alpha(\xi)^{1 / n} r(\xi)^{\prime n-1 / n}},
$$

where

$$
r(x)=\int_{0}^{x}(q(\xi)-f(\xi)) d \xi,
$$

does not have a root between 0 and $a$; otherwise we are in case $\mathrm{B}_{1}$ or $\mathrm{B}_{2}$. This is clear from (4.5) below. The prior distinction between $B_{1}$ and $B_{2}$ will appear in the discussion below. As in the discussion in Sec. 3, if $G(x)$ is the right side of (4.3) then $G(a) \leq T$ implies case A and $G(a)>T$ implies $\mathrm{B}_{1}$ or $\mathrm{B}_{2}$.

In case $\mathrm{A}$ there are three domains $D_{1}, D_{2}$, and $D_{3}: D_{2}$ and $D_{3}$ are as in case $\mathrm{A}, \mathrm{Sec}$. 2, and $D_{1}$ is the part of $D$ between $t=T$ and $t=t^{0}(x)$. The solution of (4.2)-(3.4) in $D_{2}$ is

$$
h\left(x, t_{0}\right)=\left[\frac{r(x)}{\alpha(x)}\right]^{1 / n}, \quad t\left(x, t_{0}\right)=t_{0}+\int_{0}^{x} \frac{d \xi}{n \alpha(\xi)^{1 / n} r(\xi)^{1 n-1 / n}}, \quad 0 \leq t_{0} \leq T .
$$

The curves $t\left(x, t_{0}\right)$ do not intersect in $D_{2}, t_{x}\left(x, t_{0}\right)>0, t_{x_{0}}\left(x, t_{0}\right)=1$, and

$$
h(x, t)=\left[\frac{r(x)}{\alpha(x)}\right]^{1 / n} \text {. }
$$

In $D_{3}$

$$
\begin{gathered}
h\left(x, x_{0}\right)=\left[\frac{r(x)-r\left(x_{0}\right)}{\alpha(x)}\right]^{1 / n}, \\
t\left(x, x_{0}\right)=\int_{x_{0}}^{x} \frac{d \xi}{n \alpha(\xi)^{1 / n}\left[r(\xi)-r\left(x_{0}\right)\right]^{n-1 / n}}, \quad 0 \leq x_{0} \leq a .
\end{gathered}
$$

As in Sec. 3 we impose the condition

$$
\frac{d}{d x}\left[\alpha(x)(q(x)-f(x))^{\eta}\right] \geq 0
$$

in order that $t_{x_{0}}\left(x, x_{0}\right)<0$. (4.6) holds if $\alpha, q$, and $f$ are constant. Then the curves $t=t(x$, $\left.x_{0}\right)$ do not intersect in $D_{3}$, and (4.5) defines $h(x, t)$ in $D_{3}$. The solution in $D_{1}$ is obtained by solving (4.2) with $q(x)=0$, subject to the conditions

$$
t\left(x_{0}^{*}\right)=T, \quad h\left(x_{0}^{*}\right)=\left[\frac{r\left(x_{0}^{*}\right)}{\alpha\left(x_{0}^{*}\right)}\right]^{1 / n} .
$$

The solution in $D_{1}$ is

$$
\begin{gathered}
h\left(x, x_{0}^{*}\right)=\left[\frac{p\left(x_{0}^{*}\right)-s(x)}{\alpha(x)}\right]^{1 / n}, \\
t\left(x, x_{0}^{*}\right)=T+\int_{x_{0}^{*}}^{x} \frac{d \xi}{n \alpha(\xi)^{1 / n}\left[p\left(x_{0}^{*}\right)-s(\xi)\right]^{(n-1 / n}},
\end{gathered}
$$


where

$$
s(x)=\int_{0}^{x} f(\xi) d \xi
$$

and $0 \leq x_{0}{ }^{*} \leq a$. (4.7) is valid so long as $p\left(x_{0}{ }^{*}\right)-s(x)>0$; thus the free boundary $t^{0}(x)$ is obtained by solving for $x_{0}{ }^{*}$ in $p\left(x_{0}{ }^{*}\right)-s(x)=0$ and inserting in $t=t\left(x, x_{0}{ }^{*}\right)$. We have then

$$
t^{0}(x)=T+\frac{1}{n} \int_{\psi(x)}^{x} \frac{d \xi}{\alpha(\xi)^{1 / n}[s(x)-s(\xi)]^{(n-1 / / n}},
$$

where $\psi(x)$ is the root of

$$
\int_{0}^{\psi(x)} q(\xi) d \xi=\int_{0}^{x} f(\xi) d \xi
$$

Since $q(x)>f(x)$ there is always a unique root $\psi(x)<x$ of $(4.9)$ when $0 \leq x \leq a$; $\psi(x)$ is an increasing function of $x$ and $\psi(0)=0$. It is clear from (4.7) that $t_{x_{0}} *\left(x, x_{0}{ }^{*}\right)<0$. Then the curves $t=t\left(x, x_{0}{ }^{*}\right)$ do not intersect in $D_{1}$, and therefore (4.7) defines $h(x, t)$ in $D_{1}$. The behavior of $h(x, t)$ for fixed $x$ is as in Sec. 3: $h_{t}(x, t)<0$ if $(x, t) \epsilon D_{3}, h_{t}(x, t)=0$ if $(x, t) \epsilon D_{2}$, $h_{t}(x, t)>0$ if $(x, t) \epsilon D_{1}$.

Case $\mathrm{B}_{1}$ or $\mathrm{B}_{2}$ occur when (4.3) has a root $x^{*}, 0<x^{*}<a$. To distinguish between $\mathrm{B}_{1}$ and $\mathrm{B}_{2}$ we obtain $t=t\left(x, x^{*}\right)$, the prolongation of $t=t(x, 0)$ above $t=T$ and to the right of $x=x^{*}$, by setting $x_{0}{ }^{*}=x^{*}$ in (4.7). We are in case $\mathrm{B}_{1}$ if $h\left(x, x^{*}\right)>0$ for $x^{*}<x<a$, and we are in case $\mathrm{B}_{2}$ if $h\left(\bar{x}, x^{*}\right)=0, x^{*}<\bar{x}<a$, and $h\left(x, x^{*}\right)>0$ when $x^{*}<x<\bar{x}$. Thus

$$
\int_{0}^{x *} q(\xi) d \xi>\int_{0}^{x} f(\xi) d \xi
$$

for $x^{*}<x<a$ implies case $\mathrm{B}_{1}$, and the existence of $\bar{x}, x^{*}<\bar{x}<a$, such that

$$
\int_{0}^{x *} q(\xi) d \xi=\int_{0}^{x} f(\xi) d \xi
$$

implies case $B_{2}$.

In case $\mathrm{B}_{1}$ there are four domains $D_{11}, D_{12}, D_{2}$, and $D_{3}: D_{2}, D_{3}$, and $D_{12}$ are as in case B of Sec. 3, and $D_{11}$ is the region bounded by $t=t^{0}(x), t=T, t=t\left(x, x^{*}\right)$, and $x=a$. The free boundary $t^{0}(x)$ is given by (4.8). The solution in $D_{11}$ is given by (4.7), with $0 \leq x_{0}{ }^{*} \leq$ $x^{*}$. The solution in $D_{2}$ is given by (4.4), and the solution in $D_{3}$ is given by (4.5). In $D_{12}$

$$
\begin{gathered}
h\left(x, x_{0}\right)=\left[\frac{p\left(x_{0}^{*}\right)-r\left(x_{0}\right)-s(x)}{\alpha(x)}\right]^{1 / n}, \\
t\left(x, x_{0}\right)=T+\int_{x_{0} *}^{x} \frac{d \xi}{n \alpha(\xi)^{1 / n}\left[p\left(x_{0}^{*}\right)-r\left(x_{0}\right)-s(\xi)\right]^{(n-1) / n}},
\end{gathered}
$$

where

$$
T=\int_{x_{0}}^{x_{0}^{*}} \frac{d \xi}{n \alpha(\xi)^{1 / n}\left[r(\xi)-r\left(x_{0}\right)\right]^{n-1 / / n}}
$$


Analogous to the discussion in Sec. 3, we have $t_{x_{0}}\left(x, x_{0}\right)<0$ in $D_{12}$ if we impose condition (4.6). Thus (4.10) defines $h(x, t)$ in $D_{12}$. The behavior of $h(x, t)$ for $0<x \leq x^{*}$, and also when $x^{*}<x<a$ and $(x, t) \epsilon D_{11}$ or $(x, t) \epsilon D_{3}$, is as in case $A$. When $(x, t) \epsilon D_{12}$ it can be seen from (4.10) that $h_{x_{0}}\left(x, x_{0}\right) \geq 0$, so $h_{t}(x, t)<0$.

The discussion for the case $B_{2}$ is the same as for the case $B_{1}$. In case $B_{2}$ the upper boundary of $D_{12}$ consists partly of $t\left(x, x^{*}\right)$ and partly of $t^{0}(x)$; we need an explicit determination of the latter when $x \geq \bar{x}$. This is obtained from (4.10) and (4.11): from (4.10) we get

$$
p\left(x_{0}^{*}\right)-r\left(x_{0}\right)-s(x)=0 .
$$

Then

$$
t^{0}(x)=T+\int_{\psi(x)}^{x} \frac{d \xi}{n \alpha(\xi)^{1 / n}[s(x)-s(\xi)]^{(n-1 / / n}},
$$

where $\bar{\psi}(x)=x_{0}^{*}(x)$ is obtained from (4.11) and (4.12).

We consider next

$$
((L-x) h)_{t}+\left((L-x) \alpha h^{n}\right)_{x}=(L-x)(q(x)-f(x)) .
$$

If to (3.16) we add $f(x)(L-x)=\bar{f}(x)$ then (4.14) is the same as (4.1). If we impose the condition

$$
\frac{d}{d x} \alpha(x)(L-x)(q(x)-f(x))^{n} \geq 0
$$

then the entire discussion above applies to (4.14) and (4.4). But, as in Sec. 3, condition (4.15) is unsatisfactory since it excludes the case $\alpha(x), q(x)$, and $f(x)$ constant. Again the situation in $D_{2} \cup D_{1}$ or $D_{2} \cup D_{11}$ is not affected. As in Sec. 3 we can replace $(4.15)$ by

$$
\frac{d}{d x} \frac{L-x}{\alpha(x)}<0 \quad \text { and } \quad q^{\prime}(x)-f^{\prime}(x) \geq 0 .
$$

Then (4.16) includes the case $\alpha(x), q(x), f(x)$ constant, and implies that the solution in $D_{3}$ is given by appropriately modified expressions in the discussion above. This is also true in $D_{12}$, but we omit the detailed argument.

\section{REFERENCES}

[1] P. S. Eagleson, Dynamic hydrology, McGraw-Hill, 1970

[2] B. Sherman and V. P. Singh, A distributed converging overland flow model, I and 2, Water Resources Res. 12, 889-901 (1976)

[3] R. A. Wooding, A hydraulic model for the catchment-stream problem: 1. Kinematic wave theory, J. Hydrology 3, 254-267 (1965)

[4] D. A. Woolhiser and J. A. Liggett. One-dimensional flow over a plane-the rising hydrograph. Water Resources Res. 3, 753-771 (1967)

[5] D. A. Woolhiser, Overland flow on a converging surface. Trans. ASAE 12, 460-462 (1969) 